\title{
Dynamic Analysis of an Impulsive Predator-Prey Model with Disease in Prey and Ivlev-Type Functional Response
}

\author{
Yuanfu Shao, ${ }^{1}$ Peiluan $\mathrm{Li}^{2}{ }^{2}$ and Guoqiang Tang ${ }^{1}$ \\ ${ }^{1}$ School of Science, Guilin University of Technology, Guangxi, Guilin 541004, China \\ ${ }^{2}$ School of Mathematics and Statics, Henan University of Science and Technology, Luoyang, \\ Henan 471003, China \\ Correspondence should be addressed to Yuanfu Shao, shaoyuanfu@163.com
}

Received 14 April 2012; Revised 13 May 2012; Accepted 14 May 2012

Academic Editor: Narcisa C. Apreutesei

Copyright (C) 2012 Yuanfu Shao et al. This is an open access article distributed under the Creative Commons Attribution License, which permits unrestricted use, distribution, and reproduction in any medium, provided the original work is properly cited.

\begin{abstract}
A predator-prey model with disease in prey, Ivlev-type functional response, and impulsive effects is proposed. By using Floquet theory and small amplitude perturbation skill, sufficient conditions of the existence and global stability of susceptible pest-eradication periodic solution are obtained. By impulsive comparison theorem, conditions ensuring the permanence of the system are established. Examples and simulation are given to show the complex dynamics for the key parameters.
\end{abstract}

\section{Introduction}

It is well known that many evolution processes are characterized by the fact that at certain moments their stage changes abruptly. For example, for integrated pest management (IPM) strategy on ecosystem, the predators are released periodically every time $T$, and periodic catching or spraying pesticides are also applied. Hence, the predator and prey experience a change of state abruptly. It is natural to assume that these processes act in the form of impulse. The effects of impulsion on the dynamics of predator-prey system have been investigated extensively, see [1-15].

On the other hand, in population dynamics, a functional response of the predator to the prey density refers to the change in the density of prey attached per unit time per predator as the prey density changes. Usually, functional response plays key role in the dynamics of predator-prey system [16-18]. Recently, many different functional responses are studied such as Holling-type $[8,11]$, Beddington-type $[7,12]$, and Watt-type $[13,14]$. Ivlev-type functional 
response is the most common type of functional response among arthropod predators, and much progress has been seen in the study of predator-prey model with Ivlev-type functional response [19-21].

Considering the influence of periodic pesticide spraying on all species and periodically releasing predator at fixed different time, authors [1] proposed and studied the following predator-prey system with impulsive perturbation and Ivlev-type functional response:

$$
\begin{gathered}
x^{\prime}(t)=a x(t)\left(1-\frac{x(t)}{b}\right)-h y(t)\left(1-e^{-c x(t)}\right), \quad t \neq(n+\imath-1) T, t \neq n T, \\
y^{\prime}(t)=e y(t)\left(1-e^{-c x(t)}\right)-d y(t), \\
x\left(t^{+}\right)=\left(1-p_{1}\right) x(t), \quad y\left(t^{+}\right)=\left(1-p_{2}\right) y(t), \quad t=(n+\iota-1) T, \\
x\left(t^{+}\right)=x(t), \quad y\left(t^{+}\right)=y(t)+q, \quad t=n T .
\end{gathered}
$$

They obtained the local stability of prey-free periodic solution and permanence of the system.

However, for biological control, in addition to the approach to release natural enemies, another approach is to use microbial control with pathogens since diseases can be important natural controls of some pests. For example, insects can be infected by diseasecausing organism such as bacteria and viruses. Under appropriate condition, these naturally occurring organisms may multiply to cause disease outbreak that can decimate an insect population. There is a large amount of literatures on applications of entomopathogens to suppress pests [22-26]. Then how does the disease in prey affect the dynamics of above system? Further, whether we can derive the global stability of the susceptible pest-eradication periodic solution?

Motivated by above discussion, in this paper, we are concerned with the following predator-prey model with complex influence of disease in prey, Ivlev-type functional response, and impulsive perturbation as follows:

$$
\begin{aligned}
& S^{\prime}(t)=r S(t)\left(1-\frac{S(t)+I(t)}{K}\right)-\alpha S(t) I(t)-a y(t)\left(1-e^{-c S(t)}\right), \\
& I^{\prime}(t)=\alpha S(t) I(t)-d_{1} I(t), \\
& \begin{aligned}
y^{\prime}(t)=\sigma a y(t)\left(1-e^{-c S(t)}\right)-d_{2} y(t), \\
S\left(t^{+}\right)=\left(1-p_{1}\right) S(t), \\
I\left(t^{+}\right)=\left(1-p_{2}\right) I(t), \quad t=(n+\imath-1) T, \\
y\left(t^{+}\right)=\left(1-p_{3}\right) y(t), \\
\quad S\left(t^{+}\right)=S(t), \\
I\left(t^{+}\right)=I(t)+q_{1}, \quad t=n T, \\
y\left(t^{+}\right)=y(t)+q_{2},
\end{aligned}
\end{aligned}
$$


where $S(t)$ and $I(t)$ represent densities of susceptible prey (pest) population and infective prey (pest) population, respectively; $y(t)$ is the density of predator (natural enemy), and $S\left(t^{+}\right)=\lim _{v \rightarrow t^{+}} S(v), I\left(t^{+}\right)=\lim _{v \rightarrow t^{+}} I(v), y\left(t^{+}\right)=\lim _{v \rightarrow t^{+}} y(v)$ exist.

For (1.2), we give the following biological assumptions.

(i) The growth rate for prey is $r S(t)(1-(S(t)+I(t)) / K), r>0$ is intrinsic growth rate, and $K>0$ is the carrying capacity.

(ii) There are diseases among prey population, and the prey population is divided into susceptible class and infective class. The incidence rate is classic bilinear as $\alpha S(t) I(t)$, and $\alpha$ is the contact number per unit time for every infective prey with susceptible prey such that $r / K+\alpha \leq r$.

(iii) The predator only catches susceptible prey, and the predation functional response is Ivlev type. Parameters $a, c$ are positive constants, and $\sigma$ is conversion rate from prey to predator.

(iv) Parameters $d_{1}, d_{2}$ are death rates for infective prey and predator, respectively.

(v) $0 \leq p_{i}<1(i=1,2,3)$ represents the fraction of prey and predator which due to the pesticide at $t=(n+\iota-1) T, 0<\iota<1, q_{1}, q_{2}$ are positive constants representing the release amount of infective prey and predator periodically at time $t=n T$ respectively, where $n \in Z_{+}$(positive integer set), and $T$ is the period of impulsive effect.

By using Floquet theory, comparison method of impulsive differential equation, and numerical analysis skill, we aim to study the dynamics of (1.2) with disease in prey, Ivlevtype functional response, and impulsive effects.

The rest of the paper is organized as follows. In Section 2, some preliminaries are introduced. In Section 3, by using the Floquet theory and small amplitude perturbation skill and comparison theorem of impulsive differential equation, the existence of susceptible pest-eradication periodic solution and permanence of system (1.2) are studied. In Section 4, some examples and numerical analysis are given to show the rich dynamics of (1.2). Finally, biological implications and a brief discussion are given in Section 5 to conclude this paper.

\section{Preliminaries}

Let $R_{+}=[0, \infty)$ and $R_{+}^{3}=\left\{z=\left(z_{1}, z_{2}, z_{3}\right)^{T} \in R^{3}: z_{i} \in R_{+}, i=1,2,3\right\}$. Denote by $f=$ $\left(f_{1}, f_{2}, f_{3}\right)^{T}$ the map defined by right-hand sides of the first three equations of (1.2). Let $V$ : $R_{+} \times R_{+}^{3} \rightarrow R_{+}$, and then $V$ is said to belong to class $V_{0}$ if

(i) $V$ is continuous in $((n-1) T,(n+\iota-1) T] \times R_{+}^{3}$, and $((n+\iota-1) T, n T] \times R_{+}^{3}$, $\lim _{(t, s) \rightarrow\left((n+l-1) T^{+}, z\right)} V(t, s)=V\left((n+\iota-1) T^{+}, z\right)$, and $\lim _{(t, s) \rightarrow\left(n T^{+}, z\right)} V(t, s)=V\left(n T^{+}, z\right)$ exist, and

(ii) $V$ is locally Lipschitzian in $z$.

Definition 2.1. Let $V \in V_{0}$, then for $(t, z) \in((n-1) T,(n+\iota-1) T] \times R_{+}^{3}$ and $(t, z) \in((n+\iota-$ 1) $T, n T] \times R_{+}^{3}$, the upper right derivative of $V(t, z)$ with respect to system (1.2) is defined as

$$
D^{+} V(t, z)=\lim \sup _{h \rightarrow 0^{+}} \frac{1}{h}[V((t+h), z+h f(t, z))-V(t, z)]
$$


Definition 2.2. System (1.2) is said to be permanent if there exist positive constants $m$ and $M$ with $M \geq m>0$ such that each positive solution $(S(t), I(t), y(t))$ satisfying $m \leq S(t), I(t)$, and $y(t) \leq M$ for all $t$ is sufficiently large.

The solution of system (1.2) is continuously differentiable on $((n-1) T, n+\iota-1) T]$ and $((n+\iota-1) T, n T], n \in Z_{+}$. Obviously, the global existence and uniqueness of solutions to system (1.2) are guaranteed by the smoothness properties of function $f$. For more details see [27].

Lemma 2.3. Suppose that $(S(t), I(t), y(t))$ is a solution of system (1.2) with $S\left(0^{+}\right) \geq 0, I\left(0^{+}\right) \geq 0$, $y\left(0^{+}\right) \geq 0$. Then $S(t) \geq 0, I(t) \geq 0, y(t) \geq 0$ for all $t \geq 0$. Furthermore, $S(t)>0, I(t)>0, y(t)>0$ if $S\left(0^{+}\right)>0, I\left(0^{+}\right)>0, y\left(0^{+}\right)>0$.

Lemma 2.4 (see [28]). Let $V \in V_{0}$. Assume that

$$
\begin{gathered}
D^{+} V(t, z) \leq g(t, V(t, z)), \quad t \neq n T, \quad t \neq(n+\iota-1) T, \\
V\left(t, z\left(t^{+}\right)\right) \leq \psi_{n}(V(t, z)), \quad t=n T, \\
V\left(t, z\left(t^{+}\right)\right) \leq \phi_{n+\iota}(V(t, z)), \quad t=(n+\iota-1) T,
\end{gathered}
$$

where $g: R_{+} \times R_{+} \rightarrow R$ is continuous in $((n-1) T,(n+\iota-1) T] \times R_{+}$, and $((n+\iota-1) T, n T] \times R_{+}$, $\lim _{(t, s) \rightarrow\left((n+l-1) T^{+}, z\right)} g(t, s)=g\left((n+\iota-1) T^{+}, z\right)$, and $\lim _{(t, s) \rightarrow\left(n T^{+}, z\right)} g(t, s)=g\left(n T^{+}, z\right)$ exist; $\psi_{n}:$ $R_{+} \rightarrow R_{+}$and $\phi_{n+l}: R_{+} \rightarrow R_{+}$are nondecreasing. Let $r(t)$ be the maximal solution of the scalar impulsive differential equation

$$
\begin{gathered}
\frac{d u(t)}{d t}=g(t, u(t)), \quad t \neq(n+\iota-1) T, t \neq n T, \\
u\left(t^{+}\right)=\psi_{n}(u(t)), \quad t=n T, \\
u\left(t^{+}\right)=\phi_{n+\iota}(u(t)), \quad t=(n+\iota-1) T, \\
u\left(0^{+}\right)=u_{0},
\end{gathered}
$$

existing on $[0, \infty)$. Then $V\left(0^{+}, z\left(0^{+}\right)\right) \leq u_{0}$ implies that $V(t, z(t)) \leq r(t)$ for $t \geq 0$, where $z(t)=$ $(S(t), I(t), y(t))$ is any solution of system (1.2). Assume that all the inequalities " $\leq$ " in system (2.2) are replaced by " $\geq$ " in the preceding equations, and let $\rho(t)$ be the minimal solution of (2.3) existing on $[0, \infty)$. Then $V\left(0^{+}, z\left(0^{+}\right)\right) \geq u_{0}$ implies that $V(t, z(t)) \geq \rho(t)$ for $t \geq 0$.

Lemma 2.5. There exists a positive constant $M$ such that $S(t) \leq M, I(t) \leq M, y(t) \leq M$ for each solution of system (1.2) with positive initial values, where $t$ is sufficiently large.

Proof. Define $V(t)=\sigma S(t)+\sigma I(t)+y(t)$, then it is clear that $V \in V_{0}$. For the continuity points of (1.2), that is, $t \neq(n+\iota-1) T$ and $t \neq n T$, then $D^{+} V(t)+d V(t)=V^{\prime}(t)+d V(t)$, by simple computation, we have

$$
\begin{aligned}
D^{+} V(t)+d V(t)= & \sigma(d+r) S(t)-\sigma I(t)\left(d_{1}-d\right) \\
& -\left(d_{2}-d\right) y(t)-\frac{\sigma r S(t)(S(t)+I(t))}{K}
\end{aligned}
$$


Abstract and Applied Analysis

$$
\begin{aligned}
& \leq \sigma(d+r) S(t)-\frac{\sigma r S^{2}(t)}{K} \\
& \leq M_{1}
\end{aligned}
$$

where $d=\min \left\{d_{1}, d_{2}\right\}$ and $M_{1}=\sigma K(d+r)^{2} / 4 r$.

When $t=(n+\iota-1) T$, by system (1.2) directly, we have

$$
V\left((n+\iota-1) T^{+}\right) \leq V((n+\iota-1) T) .
$$

Similarly, when $t=n T$, we have

$$
V\left(n T^{+}\right) \leq V(n T)+\sigma q_{1}+q_{2}
$$

According to Lemma 2.4, we can obtain that

$$
\begin{aligned}
V(t) & \leq\left(V\left(0^{+}\right)-\frac{M_{1}}{d}\right) e^{-d t}+\frac{\left(\sigma q_{1}+q_{2}\right) e^{d T}\left(1-e^{-d t}\right)}{e^{d T}-1}+\frac{M_{1}}{d} \\
& \longrightarrow \frac{M_{1}}{d}+\frac{\left(\sigma q_{1}+q_{2}\right) e^{d T}}{e^{d T}-1} .
\end{aligned}
$$

Thus, $V(t)$ is uniformly ultimately bounded from above. By the definition of $V(t)$, we follow the conclusion immediately. This completes the proof.

Lemma 2.6. For the following system:

$$
\begin{gathered}
u^{\prime}(t)=-d u(t), \quad t \neq(n+\imath-1) T, \quad t \neq n T, \\
u\left(t^{+}\right)=(1-p) u(t), \quad t=(n+\iota-1) T, \\
u\left(t^{+}\right)=u(t)+q, \quad t=n T .
\end{gathered}
$$

System (2.8) has a positive periodic solution $\boldsymbol{u}^{*}(t)$ and for every solution $u(t)$ of system (2.8), $\mid u(t)-$ $u^{*}(t) \mid \rightarrow 0$ as $t \rightarrow \infty$, where

$$
\begin{gathered}
u^{*}(t)=\left\{\begin{array}{l}
\frac{q e^{-d(t-(n-1) T)}}{1-(1-p) e^{-d T}}, \quad(n-1) T<t \leq(n+\iota-1) T, \\
\frac{q(1-p) e^{-d(t-(n-1) T)}}{1-(1-p) e^{-d T}}, \quad(n+\iota-1) T<t \leq n T,
\end{array}\right. \\
u^{*}\left(0^{+}\right)=u^{*}\left(n T^{+}\right)=\frac{q}{1-(1-p) e^{-d T}}, \\
u^{*}\left((n+\iota-1) T^{+}\right)=u^{*}\left(n T^{+}\right)=\frac{q(1-p) e^{-d i T}}{1-(1-p) e^{-d T}} .
\end{gathered}
$$


Proof. It is easily verified that $u^{*}(t)$ is a periodic solution of system (2.8) with the given initial values. For the solution $u(t)$ of system (2.8), we can derive that

$$
u(t)= \begin{cases}(1-p)^{n-1}\left(u\left(0^{+}\right)-u^{*}\left(0^{+}\right)\right) e^{-d t}+u^{*}(t), & t \in((n-1) T,(n+\iota-1) T], \\ (1-p)^{n}\left(u\left(0^{+}\right)-u^{*}\left(0^{+}\right)\right) e^{-d t}+u^{*}(t), & t \in((n+\iota-1) T, n T] .\end{cases}
$$

Therefore, $\lim _{t \rightarrow \infty}\left|u(t)-u^{*}(t)\right|=0$. This completes the proof.

\section{Extinction and Permanence}

For (1.2), if $S(t) \equiv 0$ for all $t \geq 0$, we have the following subsystem of (1.2):

$$
\begin{gathered}
I^{\prime}(t)=-d_{1} I(t), \quad t \neq(n+\imath-1) T, \quad t \neq n T, \\
y^{\prime}(t)=-d_{2} y(t), \\
I\left(t^{+}\right)=\left(1-p_{2}\right) I(t), \quad y\left(t^{+}\right)=\left(1-p_{3}\right) y(t), \quad t=(n+\iota-1) T, \\
I\left(t^{+}\right)=I(t)+q_{1}, \quad y\left(t^{+}\right)=y(t)+q_{2}, \quad t=n T .
\end{gathered}
$$

For subsystem (3.1), by assumption (iii), there is no relation between $I(t)$ and $y(t)$. By Lemma 2.6, we have the following conclusion.

Lemma 3.1. System (3.1) has a unique positive periodic solution

$$
\begin{gathered}
I^{*}(t)= \begin{cases}\frac{q_{1} e^{-d_{1}(t-(n-1) T)}}{1-\left(1-p_{2}\right) e^{-d_{1} T},} & (n-1) T<t \leq(n+\iota-1) T, \\
\frac{q_{1}\left(1-p_{2}\right) e^{-d_{1}(t-(n-1) T)}}{1-\left(1-p_{2}\right) e^{-d_{1} T}}, & (n+\iota-1) T<t \leq n T,\end{cases} \\
y^{*}(t)= \begin{cases}\frac{q_{2} e^{-d_{2}(t-(n-1) T)}}{1-\left(1-p_{3}\right) e^{-d_{2} T}}, & (n-1) T<t \leq(n+\iota-1) T, \\
\frac{q_{2}\left(1-p_{3}\right) e^{-d_{2}(t-(n-1) T)}}{1-\left(1-p_{3}\right) e^{-d_{2} T}}, & (n+\iota-1) T<t \leq n T,\end{cases}
\end{gathered}
$$

with initial values

$$
\begin{gathered}
I^{*}\left(0^{+}\right)=I^{*}\left(n T^{+}\right)=\frac{q_{1}}{1-\left(1-p_{2}\right) e^{-d_{1} T}}, \\
I^{*}\left((n+\iota-1) T^{+}\right)=I^{*}\left(n T^{+}\right)=\frac{q_{1}\left(1-p_{2}\right) e^{-d_{1} l T}}{1-\left(1-p_{2}\right) e^{-d_{1} T}},
\end{gathered}
$$


Abstract and Applied Analysis

$$
\begin{gathered}
y^{*}\left(0^{+}\right)=y^{*}\left(n T^{+}\right)=\frac{q_{2}}{1-\left(1-p_{3}\right) e^{-d_{2} T}}, \\
y^{*}\left((n+\iota-1) T^{+}\right)=y^{*}\left(n T^{+}\right)=\frac{q_{2}\left(1-p_{3}\right) e^{-d_{2} L T}}{1-\left(1-p_{3}\right) e^{-d_{2} T}} .
\end{gathered}
$$

Next, we investigate the stability of the susceptible pest-eradication periodic solution $\left(0, I^{*}(t), y^{*}(t)\right)$ of system (1.2).

Theorem 3.2. Let $(S(t), I(t), y(t))$ be any solution of $(1.2)$. Then $\left(0, I^{*}(t), y^{*}(t)\right)$ is globally asymptotically stable provided that

$$
\begin{aligned}
\left(H_{1}\right): r T & -\left(\frac{r}{K}+\alpha\right) \frac{q_{1}\left[\left(1-p_{2}\right) e^{-d_{1} T}+p_{2} e^{-d_{1} T T}-1\right]}{d_{1}\left[\left(1-p_{2}\right) e^{-d_{1} T}-1\right]} \\
& -a c \frac{q_{2}\left[\left(1-p_{3}\right) e^{-d_{2} T}+p_{3} e^{-d_{2} T}-1\right]}{d_{2}\left[\left(1-p_{3}\right) e^{-d_{2} T}-1\right]}<\ln \frac{1}{1-p_{1}} .
\end{aligned}
$$

Proof. First, by using Floquet theory, we show the local stability of periodic solution $\left(0, I^{*}(t), y^{*}(t)\right)$ of $(1.2)$. Considering the behavior of small amplitude perturbation, let

$$
S(t)=u(t), \quad I(t)=v(t)+I^{*}(t), \quad y(t)=w(t)+y^{*}(t),
$$

where $u(t), v(t)$, and $w(t)$ are all small perturbations. By using Taylor expansion and after neglecting higher-order terms, (1.2) can be linearized, and the linearized equations read as

$$
\begin{gathered}
u^{\prime}(t)=\left(r-\frac{r I^{*}(t)}{K}-\alpha I^{*}(t)-a c y^{*}(t)\right) u(t), \\
v^{\prime}(t)=\alpha I^{*}(t) u(t)-d_{1} v(t), \quad t \neq(n+\imath-1) T, t \neq n T, \\
w^{\prime}(t)=-a c \sigma y^{*}(t) u(t)-d_{2} w(t), \\
u\left(t^{+}\right)=\left(1-p_{1}\right) u(t), \quad v\left(t^{+}\right)=\left(1-p_{2}\right) v(t), \quad w(t)=\left(1-p_{3}\right) w(t), \quad t=(n+\iota-1) T, \\
u\left(t^{+}\right)=u(t), \quad v\left(t^{+}\right)=v(t)+q_{1}, \quad w\left(t^{+}\right)=w(t)+q_{2}, \quad t=n T .
\end{gathered}
$$

Suppose that $\Phi(t)$ is the fundamental solution matrix of system (3.6), and then $\Phi(t)$ satisfies

$$
\frac{d \Phi(t)}{d t}=\left(\begin{array}{ccc}
r-\frac{r I^{*}(t)}{K}-\alpha I^{*}(t)-a c y^{*}(t) & 0 & 0 \\
\alpha I^{*}(t) & -d_{1} & 0 \\
a c \sigma y^{*}(t) & 0 & -d_{2}
\end{array}\right) \Phi(t)
$$


$\Phi(0)=I_{3}$ is the identical matrix. Then the resetting impulsive conditions of (1.2) become

$$
\begin{gathered}
\left(\begin{array}{c}
u\left((n+\iota-1) T^{+}\right) \\
v\left((n+\iota-1) T^{+}\right) \\
w\left((n+\iota-1) T^{+}\right)
\end{array}\right)=\left(\begin{array}{ccc}
1-p_{1} & 0 & 0 \\
0 & 1-p_{2} & 0 \\
0 & 0 & 1-p_{3}
\end{array}\right)\left(\begin{array}{c}
u((n+\iota-1) T) \\
v((n+\iota-1) T) \\
w((n+\iota-1)) T
\end{array}\right), \\
\left(\begin{array}{c}
u\left(n T^{+}\right) \\
v\left(n T^{+}\right) \\
w\left(n T^{+}\right)
\end{array}\right)=\left(\begin{array}{lll}
1 & 0 & 0 \\
0 & 1 & 0 \\
0 & 0 & 1
\end{array}\right)\left(\begin{array}{c}
u(n T) \\
v(n T) \\
w(n T)
\end{array}\right) .
\end{gathered}
$$

By Floquet theory, the local stability of $\left(0, I^{*}(t), y^{*}(t)\right)$ is determined by the eigenvalues of

$$
N=\left(\begin{array}{ccc}
1-p_{1} & 0 & 0 \\
0 & 1-p_{2} & 0 \\
0 & 0 & 1-p_{3}
\end{array}\right)\left(\begin{array}{lll}
1 & 0 & 0 \\
0 & 1 & 0 \\
0 & 0 & 1
\end{array}\right) \Phi(t)
$$

where

$$
\Phi(t)=\left(\begin{array}{ccc}
\left(1-p_{1}\right) e^{\int_{0}^{T}\left(r-r I^{*}(t) / K-\alpha I^{*}(t)-a c y^{*}(t)\right) d t} & 0 & 0 \\
* & \left(1-p_{2}\right) e^{-d_{1} T} & 0 \\
* * & 0 & \left(1-p_{3}\right) e^{-d_{2} T}
\end{array}\right) .
$$

The exact expression of $*$ and $* *$ are omitted since they are not required in the analysis that follows. The eigenvalues of $N$ are

$$
\lambda_{1}=\left(1-p_{1}\right) e^{\int_{0}^{T}\left(r-r I^{*}(t) / K-\alpha I^{*}(t)-a c y^{*}(t)\right) d t}, \quad \lambda_{2}=\left(1-p_{2}\right) e^{-d_{1} T}, \quad \lambda_{3}=\left(1-p_{3}\right) e^{-d_{2} T}
$$

Obviously, $\lambda_{2}<1, \lambda_{3}<1$. It follows that $\left|\lambda_{1}\right|<1$ if and only if

$$
r T-\int_{0}^{T}\left(\frac{r I^{*}(t)}{K}+\alpha I^{*}(t)+a c y^{*}(t)\right) d t<\ln \frac{1}{1-p_{1}} .
$$

By computation, we have

$$
\begin{aligned}
& \int_{0}^{T} I^{*}(t) d t=\frac{q_{1}\left(\left(1-p_{2}\right) e^{-d_{1} T}+p_{2} e^{-d_{1} t T}-1\right)}{d_{1}\left(\left(1-p_{2}\right) e^{-d_{1} T}-1\right)} \\
& \int_{0}^{T} y^{*}(t) d t=\frac{q_{2}\left(\left(1-p_{3}\right) e^{-d_{2} T}+p_{3} e^{-d_{2} t T}-1\right)}{d_{2}\left(\left(1-p_{3}\right) e^{-d_{2} T}-1\right)} .
\end{aligned}
$$

Therefore, we can derive that $\left|\lambda_{1}\right|<1$ if and only if $\left(H_{1}\right)$ holds true. Thus, by Floquet theory, under condition $\left(H_{1}\right)$, the positive periodic solution $\left(0, I^{*}(t), y^{*}(t)\right)$ is locally asymptotically stable. 
Next, we prove the globally attractive property.

Choose $\varepsilon_{1}>0$ such that

$$
\left(1-p_{1}\right) \exp \left\{\int_{0}^{T}\left(r-\left(\frac{r}{K}+\alpha\right)\left(I^{*}(t)-\varepsilon_{1}\right)-a c\left(y^{*}(t)-\varepsilon_{1}\right)\right) d t\right\} \triangleq \delta<1
$$

Besides, we have

$$
I^{\prime}(t)=\alpha S(t) I(t)-d_{1} I(t) \geq-d_{1} I(t)
$$

From Lemma 3.1 and comparison theorem of impulsive equation, for $t$ sufficiently large, we have

$$
I(t) \geq I^{*}(t)-\varepsilon_{1}
$$

Similarly, for $t$ sufficiently large, we have

$$
y(t) \geq y^{*}(t)-\varepsilon_{1}
$$

For simplification, we suppose that (3.16) and (3.17) hold for all $t \geq 0$. Hence, it follows that

$$
\begin{gathered}
S^{\prime}(t)=r S(t)\left(1-\frac{S(t)+I(t)}{K}\right)-\alpha S(t) I(t)-a y(t)\left(1-e^{-c S(t)}\right) \\
\leq S(t)\left(r-\left(\frac{r}{K}+\alpha\right)\left(I^{*}(t)-\varepsilon_{1}\right)-a c\left(y^{*}(t)-\varepsilon_{1}\right)\right), \quad t \neq(n+\iota-1) T, \\
S\left(t^{+}\right)=\left(1-p_{1}\right) S(t), \quad t=(n+\iota-1) T .
\end{gathered}
$$

Integrating (3.18) on $((n+\iota-1) T),(n+\iota) T]$ leads to

$$
\begin{aligned}
S((n+\iota) T) \leq & S\left((n+\iota-1) T^{+}\right) \exp \left\{\int_{(n+\iota-1) T}^{(n+\iota) T}\left(r-\left(\frac{r}{K}+\alpha\right)\left(I^{*}(t)-\varepsilon_{1}\right)-a c\left(y^{*}(t)-\varepsilon_{1}\right)\right) d t\right\} \\
= & S((n+\iota-1) T)\left(1-p_{1}\right) \\
& \times \exp \left\{\int_{(n+\iota-1) T}^{(n+\iota) T}\left(r-\left(\frac{r}{K}+\alpha\right)\left(I^{*}(t)-\varepsilon_{1}\right)-a c\left(y^{*}(t)-\varepsilon_{1}\right)\right) d t\right\} \\
= & S((n+\iota-1) T) \delta .
\end{aligned}
$$

Thus $S((n+\iota) T) \leq S(\iota T) \delta^{n}$. In virtue of the assumption $\delta<1$, we can follow that $S((n+\iota) T) \rightarrow$ 0 as $n \rightarrow \infty$. Noting that $0<S(t)<S((n+\iota-1) T)\left(1-p_{1}\right) e^{r T}$ for $(n+\iota-1) T \leq t \leq(n+\iota) T$, hence $S(t) \rightarrow 0$ as $n \rightarrow \infty$. 
Next, we prove $I(t) \rightarrow I^{*}(t)$ as $t \rightarrow \infty$. For an arbitrary positive constant $\varepsilon$ small enough such that $0<\varepsilon<d_{1} / \alpha$, since $0<S(t)<\varepsilon$ for $t$ large enough, without loss of generality, we assume that $0<S(t)<\varepsilon$ hold for all $t \geq 0$. Then

$$
-d_{1} I(t) \leq I^{\prime}(t)=\alpha S(t) I(t)-d_{1} I(t) \leq\left(\alpha \varepsilon-d_{1}\right) I(t) .
$$

By Lemma 3.1 and comparison theorem again, for all $\varepsilon^{\prime}>0$, there exists $\bar{T}>0$, for all $t>\bar{T}$, and we obtain

$$
I^{*}(t)-\varepsilon^{\prime} \leq I(t) \leq I^{\Delta}(t)+\varepsilon^{\prime},
$$

where $I^{*}(t)$ is defined in Lemma 3.1, and $I^{\Delta}(t)$ is the solution of the following system:

$$
\begin{gathered}
I(t)=\left(\alpha \varepsilon-d_{1}\right) I(t), \quad t \neq(n+\iota-1) T, \quad t \neq n T, \\
I\left(t^{+}\right)=\left(1-p_{2}\right) I(t), \quad t=(n+\iota-1) T, \\
I\left(t^{+}\right)=I(t)+q_{1}, \quad t=n T,
\end{gathered}
$$

that is,

$$
I^{\Delta}(t)= \begin{cases}\frac{q_{1} e^{\left(-d_{1}+\alpha \varepsilon\right)(t-(n-1) T)}}{1-\left(1-p_{2}\right) e^{\left(-d_{1}+\alpha \varepsilon\right) T}}, & (n-1) T<t \leq(n+\iota-1) T \\ \frac{q_{1}\left(1-p_{2}\right) e^{\left(-d_{1}+\alpha \varepsilon\right)(t-(n-1) T)}}{1-\left(1-p_{2}\right) e^{\left(-d_{1}+\alpha \varepsilon\right) T}}, & (n+\iota-1) T<t \leq n T .\end{cases}
$$

Let $\varepsilon \rightarrow 0$, it follows that

$$
I^{*}(t)-\varepsilon^{\prime}<I(t)<I^{*}(t)+\varepsilon^{\prime}
$$

holds for $t$ sufficiently large. Note that $\varepsilon^{\prime}>0$ is a constant small enough, then letting $\varepsilon^{\prime} \rightarrow 0$, and we have $I(t) \rightarrow I^{*}(t)$ as $t \rightarrow \infty$. By the same method, we can similarly derive that $y(t) \rightarrow y^{*}(t)$ as $t \rightarrow \infty$. This completes the proof.

Finally, we study the permanence of system (1.2).

Theorem 3.3. System (1.2) is permanent if

$$
\begin{aligned}
\left(H_{2}\right): r T & -\left(\frac{r}{K}+\alpha\right) \frac{q_{1}\left(\left(1-p_{2}\right) e^{-d_{1} T}+p_{2} e^{-d_{1} T T}-1\right)}{d_{1}\left(\left(1-p_{2}\right) e^{-d_{1} T}-1\right)} \\
& -a c \frac{q_{2}\left(\left(1-p_{3}\right) e^{-d_{2} T}+p_{3} e^{-d_{2} L}-1\right)}{d_{2}\left(\left(1-p_{3}\right) e^{-d_{2} T}-1\right)}>\ln \frac{1}{1-p_{1}} .
\end{aligned}
$$

Proof. By Lemma 2.5, without loss of generality, we suppose that $S(t) \leq M, I(t) \leq M$ and $y(t) \leq M$ for all $t \geq 0$, where $M$ is a constant satisfying $M>r / a c$. 
On the other hand, from (3.16) and (3.17), we can obtain

$$
\begin{aligned}
& I(t) \geq I^{*}(t)-\varepsilon_{1} \geq I^{*}\left(0^{+}\right)\left(1-p_{2}\right) e^{-d_{1} T}-\varepsilon_{1} \triangleq m_{1}>0, \\
& y(t) \geq y^{*}(t)-\varepsilon \geq y^{*}\left(0^{+}\right)\left(1-p_{3}\right) e^{-d_{2} T}-\varepsilon_{1} \triangleq m_{2}>0
\end{aligned}
$$

for $t$ large enough. Therefore, $I(t)$ and $y(t)$ are ultimately positively bounded from below. Hence, we only need to prove that there exists a constant $m_{3}>0$ such that $S(t)>m_{3}$ for $t$ sufficiently large. By the proof of Theorem 3.2, assumption $\left(H_{2}\right)$ is equivalent to

$$
\int_{0}^{T}\left(r-\frac{r I^{*}(t)}{K}-\alpha I^{*}(t)-a c y^{*}(t)\right) d t>\ln \frac{1}{1-p_{1}}
$$

By the density of real number, we can select positive constant $\varepsilon$ and $\zeta$ small enough with $0<\zeta<\min \left\{d_{1} / \alpha, d_{2} / a c \sigma\right\}$ such that

$$
\eta \triangleq\left(1-p_{1}\right) e^{\int_{0}^{T}(r-r \zeta / K-(r / K+\alpha)(\bar{I}(t)+\varepsilon)-a c(\bar{y}(t)+\varepsilon)) d t}>1,
$$

where

$$
\begin{gathered}
\bar{I}(t)= \begin{cases}\frac{q_{1} e^{\left(-d_{1}+\alpha \zeta\right)(t-(n-1) T)}}{1-\left(1-p_{2}\right) e^{\left(-d_{1}+\alpha \xi\right) T},} & (n-1) T<t \leq(n+\iota-1) T, \\
\frac{q_{1}\left(1-p_{2}\right) e^{\left(-d_{1}+\alpha \zeta\right)(t-(n-1) T)}}{1-\left(1-p_{2}\right) e^{\left(-d_{1}+\alpha \zeta\right) T},} & (n+\iota-1) T<t \leq n T,\end{cases} \\
\bar{y}(t)= \begin{cases}\frac{q_{2} e^{\left(-d_{2}+a c \sigma \zeta\right)(t-(n-1) T)}}{1-\left(1-p_{3}\right) e^{\left(-d_{2}+a c \sigma \zeta\right) T}}, & (n-1) T<t \leq(n+\iota-1) T, \\
\frac{q_{2}\left(1-p_{3}\right) e^{\left(-d_{2}+a c \sigma \zeta\right)(t-(n-1) T)}}{1-\left(1-p_{3}\right) e^{\left(-d_{2}+a c \sigma \zeta\right) T}}, & (n+\iota-1) T<t \leq n T .\end{cases}
\end{gathered}
$$

We claim that $S(t)<\zeta$ cannot hold for all $t \geq 0$. Otherwise, we can follow from (1.2) that

$$
\begin{gathered}
I^{\prime}(t)=\alpha S(t) I(t)-d_{1} I(t) \leq\left(\alpha \zeta-d_{1}\right) I(t), \quad t \neq(n+\iota-1) T, \quad t \neq n T, \\
I\left(t^{+}\right)=\left(1-p_{2}\right) I(t), \quad t=(n+\iota-1) T, \\
I\left(t^{+}\right)=I(t)+q_{1}, \quad t=n T, \\
y^{\prime}(t)=\sigma a y(t)\left(1-e^{-c S(t)}\right)-d_{2} y(t) \leq\left(a c \sigma \zeta-d_{2}\right) y(t), \quad t \neq(n+\iota-1) T, t \neq n T, \\
y\left(t^{+}\right)=\left(1-p_{3}\right) y(t), \quad t=(n+\iota-1) T, \\
y\left(t^{+}\right)=y(t)+q_{2}, \quad t=n T .
\end{gathered}
$$


By comparison theorem of impulsive differential equation, then there exists a $T_{1}>0$ such that

$$
I(t) \leq \bar{I}(t)+\varepsilon, \quad y(t) \leq \bar{y}(t)+\varepsilon, \quad \forall t \geq 0,
$$

where $\bar{I}(t), \bar{y}(t)$ are defined in (3.29) and (3.30), respectively; that is, they are the solutions of systems (3.31) and (3.32), respectively. Then, for $t>T_{1}$, we have

$$
\begin{gathered}
S^{\prime}(t) \geq S(t)\left(r-\frac{r \zeta}{K}-\left(\frac{r}{K}+\alpha\right)(\bar{I}(t)+\varepsilon)-a c(\bar{y}(t)+\varepsilon)\right), \quad t \neq(n+\iota-1) T, \quad t \neq n T, \\
S\left(t^{+}\right)=\left(1-p_{1}\right) S(t), \quad t=(n+\iota-1) T \\
S\left(t^{+}\right)=S(t), \quad t=n T .
\end{gathered}
$$

Let $n_{1} \in Z_{+}$and $\left(n_{1}+\iota-1\right) T>T_{1}$. Integrating (3.34) on $((n+\iota-1) T,(n+\iota) T), n \geq n_{1}$, we have

$$
\begin{aligned}
S(n+\iota) T \geq & S\left((n+\iota-1) T^{+}\right) \exp \left\{\int_{(n+\iota-1) T}^{(n+\iota) T}\left(r-\frac{r \zeta}{K}-\left(\frac{r}{K}+\alpha\right)(\bar{I}(t)+\varepsilon)-a c(\bar{y}(t)+\varepsilon)\right)\right\} \\
= & S(n+\iota-1) T\left(1-p_{1}\right) \\
& \times \exp \left\{\int_{(n+\imath-1) T}^{(n+\iota) T}\left(r-\frac{r \zeta}{K}-\left(\frac{r}{K}+\alpha\right)(\bar{I}(t)+\varepsilon)-a c(\bar{y}(t)+\varepsilon)\right)\right\} \\
= & S(n+\iota-1) T \eta .
\end{aligned}
$$

Thus, $S\left(\left(n_{1}+n+\iota\right) T\right) \geq S\left(n_{1}+\iota\right) T \cdot \eta^{n} \rightarrow \infty$ as $t \rightarrow \infty$, which is a contradiction to the boundedness of $S(t)$. Hence, there exists a $t_{1}>0$ such that $S\left(t_{1}\right) \geq \zeta$. Then there are two cases. Case $1\left(S(t) \geq \zeta\right.$ for all $\left.t \geq t_{1}\right)$. Then let $m_{3}=\zeta$, our aim is obtained. Otherwise, we consider Case 2.

Case 2. We consider those solutions which leave the region $R=\left\{(S(t), I(t), y(t)) \in R_{+}^{3}\right.$ : $S(t)<\zeta\}$ and reenter it again. Let $t^{*}=\inf _{t \geq t_{1}}\{S(t)>\zeta\}$, then there are two possible cases for $t^{*}$.

Subcase $2.1\left(t^{*}=\left(n_{1}+\iota-1\right) T, n_{1} \in Z_{+}\right)$. Then for $t \in\left[t_{1}, t^{*}\right], S(t) \geq \zeta$ and $\left(1-p_{1}\right) \zeta \leq$ $S\left(t^{*+}\right)=\left(1-p_{1}\right) S\left(t^{*}\right)<\zeta$. Select $n_{2}, n_{3} \in Z_{+}$such that

$$
\begin{aligned}
&\left(n_{2}-1\right) T>\frac{\ln \left(\varepsilon /\left(M+q_{1}\right)\right)}{\alpha \zeta-d_{1}}, \\
&\left(1-p_{1}\right)^{n_{2}} e^{n_{2} \eta_{1} T} \eta^{n_{3}}>\left(1-p_{1}\right)^{n_{2}} e^{\left(n_{2}+1\right) \eta_{1} T} \eta^{n_{3}}>1,
\end{aligned}
$$

where $\eta_{1}=r-r \zeta / K-(r / K+\alpha) M-a c M<0$. 
Let $\widetilde{T}=n_{2} T+n_{3} T$, then we claim that there must exist a $t_{2} \in\left(t^{*}, t^{*}+\widetilde{T}\right)$ such that $S\left(t_{2}\right)>\zeta$. Otherwise, considering system (3.31), we can derive that

$$
I(t)=\left\{\begin{array}{c}
\left(1-p_{2}\right)^{n-\left(n_{1}+1\right)}\left(I\left(n_{1} T^{+}\right)-\frac{q_{1}}{1-\left(1-p_{2}\right) e^{\left(-d_{1}+\alpha \varepsilon\right) T}}\right) e^{\left(-d_{1}+\alpha \xi\right)\left(t-n_{1} T\right)}+\bar{I}(t), \\
\left(1-p_{2}\right)^{n-n_{1}}\left(I\left(n_{1} T^{+}\right)-\frac{q_{1}}{1-\left(1-p_{2}\right) e^{\left(-d_{1}+\alpha \varepsilon\right) T}}\right) e^{\left(-d_{1}+\alpha \zeta\right)\left(t-n_{1} T\right)}+\bar{I}(t), \\
(n+\iota-1) T<t \leq n T,
\end{array}\right.
$$

and $n_{1}+1 \leq n \leq n_{1}+n_{2}+n_{3}$.

From (3.36) and (3.38), we have

$$
|I(t)-\bar{I}(t)|<\left(M+q_{1}\right) e^{\left(\alpha \zeta-d_{1}\right)\left(t-n_{1} T\right)}<\varepsilon
$$

That is,

$$
I(t) \leq \bar{I}(t)+\varepsilon, \quad\left(n_{1}+n_{2}-1\right) T \leq t \leq t^{*}+\widetilde{T}
$$

Similarly, we can derive that

$$
y(t) \leq \bar{y}(t)+\varepsilon, \quad \text { for }\left(n_{1}+n_{2}-1\right) T \leq t \leq t^{*}+\tilde{T},
$$

which implies that (3.34) holds for $t^{*}+n_{2} T \leq t \leq t^{*}+\widetilde{T}$. In view of the discussion after (3.34), we have $S\left(t^{*}+\widetilde{T}\right) \geq S\left(t^{*}+n_{2} T\right) \eta^{n_{3}}$. Integrating the following system (3.42) on $\left[t^{*}, t^{*}+n_{2} T\right]$,

$$
\begin{gathered}
S^{\prime}(t) \geq S(t)\left(r-\frac{r \zeta}{K}-\left(\frac{r}{K}+\alpha\right) M-a c M\right), \quad t \neq(n+\iota-1) T, \\
S\left(t^{+}\right)=\left(1-p_{1}\right) S(t), \quad t=(n+\iota-1) T,
\end{gathered}
$$

we have

$$
S\left(t^{*}+n_{2} T\right) \geq \zeta\left(1-p_{1}\right)^{n_{2}} e^{n_{2} \eta_{1} T} .
$$

It follows from (3.37) and (3.43) that

$$
S\left(t^{*}+\tilde{T}\right) \geq \zeta\left(1-p_{1}\right)^{n_{2}} e^{n_{2} \eta_{1} T} \eta^{n_{3}}>\zeta,
$$

which is a contradiction to the above assumption, then our claim is true.

Let $\bar{t}=\inf _{t \geq t^{*}}\{S(t)>\zeta\}$, then for $t \in\left(t^{*}, \bar{t}\right), x(t) \leq \zeta, x(\bar{t})=\zeta$. Therefore,

$$
S(t) \geq \zeta\left(1-p_{1}\right)^{n_{2}+n_{3}} e^{\left(n_{2}+n_{3}\right) \eta_{1} T} \triangleq m_{3} .
$$


For $t>\bar{t}$, the same argument can be continued since $S(\bar{t}) \geq \zeta$. Subcase $2.2\left(t^{*} \neq\left(n_{1}+\iota-1\right) T, n_{1} \in Z_{+}\right)$. Then $S(t) \geq \zeta$ for $t \in\left[t_{1}, t^{*}\right]$ and $S\left(t^{*}\right)=\zeta$.

Suppose $t^{*} \in\left(\left(n_{1}^{\prime}+\iota-1\right) T,\left(n_{1}^{\prime}+\iota\right) T\right), n_{1}^{\prime} \in Z_{+}$. Then either $S(t) \leq \zeta$ for all $t \in\left(t^{*},\left(n_{1}^{\prime}+\iota\right) T\right)$, or there exists a $t \in\left(t^{*},\left(n_{1}^{\prime}+\iota\right) T\right)$ such that $S(t)>\zeta$.

If $S(t) \leq \zeta$ for all $t \in\left(t^{*},\left(n_{1}^{\prime}+\iota\right) T\right)$, similar to the former discussion, there must be a $t_{2}^{\prime} \in\left[\left(n_{1}^{\prime}+\iota\right) T,\left(n_{1}^{\prime}+\iota\right) T+\tilde{T}\right]$ such that $S\left(t_{2}^{\prime}\right)>\zeta$. Let $\tilde{t}=\inf _{t>t^{*}}\{S(t)>\zeta\}$, and then $S(\tilde{t}) \leq \zeta$ for $t \in\left(t^{*}, \tilde{t}\right]$. Therefore, $S(t) \geq \zeta\left(1-p_{1}\right)^{n_{2}+n_{3}} e^{\left(n_{2}+n_{3}+1\right) \eta_{1} T} \triangleq m_{3}$. That is, $S(t) \geq m_{3}$ holds for $t \in\left(t^{*}, \tilde{t}\right]$. For $t>\tilde{t}$, due to $S(\widetilde{t}) \geq \zeta$, the same argument can be continued.

If there exists a $t \in\left(t^{*},\left(n_{1}^{\prime}+\iota\right) T\right)$ such that $S(t)>\zeta$, the same argument in Subcase 2.1 can also be continued, and one can follow the conclusion easily. We omit it here.

Incorporating all the cases above, we deduce that system (1.2) is permanent. The proof is complete.

Remark 3.4. For system (1.2), if there is no disease in prey, then Theorems 3.2 and 3.3 reduce to the corresponding results of [1], while Theorems 3.2 and 3.3 imply that disease in prey affects dynamics of (1.2), which will be shown by simulation in Section 4 . It is interesting and valuable for biological control. Specially, the globally asymptotical stability of susceptible pest-eradication periodic solution is studied here, but authors [1] only give locally asymptotical stability of the prey-free periodic solution. Therefore, we improve and generalize the main results of [1].

\section{Examples and Simulation}

Theorems 3.2 and 3.3 show that dynamics of system(1.2) is affected by complicated factors such as contact number per unit time for every infective prey $\alpha$, impulsive period $T$, releasing amount of infective prey $q_{1}$, and releasing amount of predator $q_{2}$. In this section, by numerical analysis, we show the effects of parameters $\alpha, T, q_{1}$, and $q_{2}$ on dynamics of (1.2), respectively. For example, take $r=4, k=8, a=2, c=0.5, p_{1}=0.5, p_{2}=p_{3}=0, q_{1}=q_{2}=2, d_{1}=0.2$, $d_{2}=0.4, T=4$, and $S(0)=I(0)=y(0)=2$. If $\alpha=0.55$, then Theorem 3.2 implies that system (1.2) has a susceptible pest-eradication periodic solution, which is globally asymptotically stable. If $\alpha=0.4$, then Theorem 3.3 implies that (1.2) is permanent. By simulation, the results can be seen directly, see Figures 1 and 2, respectively. Actually, by simulation, there exists a critical value $\alpha \approx 0.531$, and if $\alpha<0.531$, then the system is permanent; otherwise, it has a susceptible pest-eradication periodic solution, see Figures 3,4 , and 5 , respectively.

Take $r=2, K=4, a=2, c=0.5, \alpha=0.6, p_{1}=0.5, p_{2}=0.3, p_{3}=0.6, \sigma=0.3, \iota=$ $0.5, d_{1}=0.2, d_{2}=0.4$, and $S(0)=I(0)=y(0)=1$. For impulsive period $T$, the releasing amount of infective prey $q_{1}$, and releasing amount of predator $q_{2}$, respectively, Theorems 3.2 and 3.3 imply that system (1.2) exhibits a variety of dynamic behaviors such as cycles, periodic doubling cascade, chaos, and so on. Take susceptible pest as an example, and the numerical analysis of susceptible pest about parameters $T, q_{1}$, and $q_{2}$ shows that dynamical behaviors of the system are very complex, see Figures 6, 7, and 8. Similarly, dynamics of infective pest and predator may be complex too. It is omitted.

\section{Discussion}

In this paper, by using Floquet theory and small amplitude perturbation technique of impulsive differential equation, we consider a predator-prey model with complex influence of disease in prey, Ivlev-type functional response, and impulsive perturbation such as 

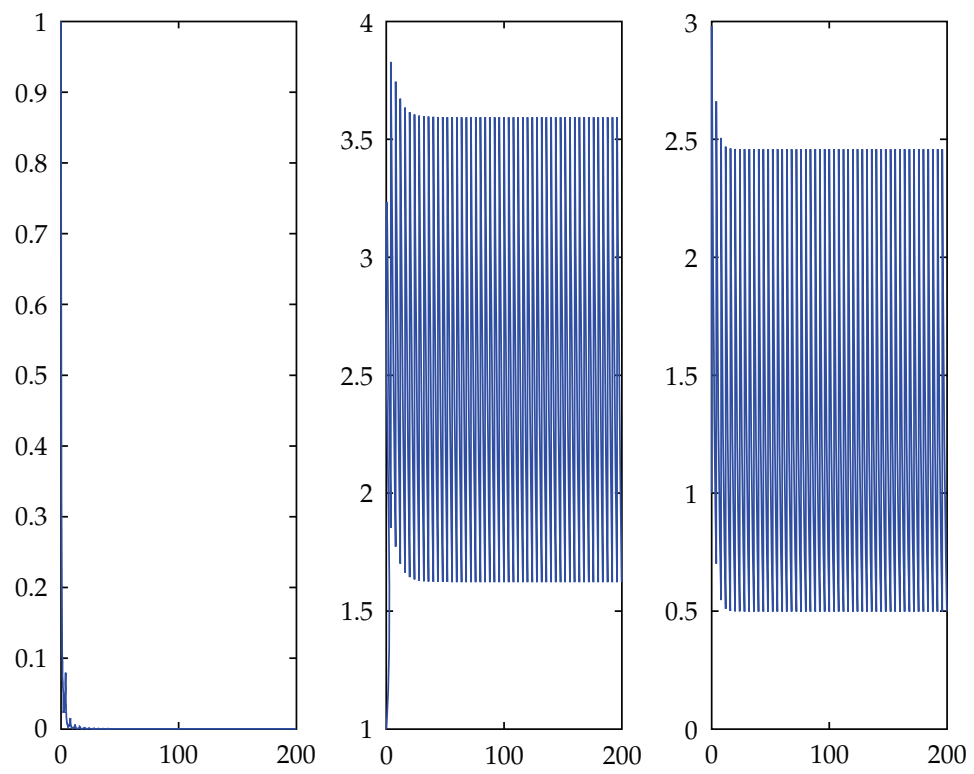

$-S(t)$

$-I(t)$

$-y(t)$

Figure 1: Existence of the pest-eradication periodic solution of (1.2) with $r=4, k=8, a=2, c=0.5, p_{1}=0.5$, $p_{2}=p_{3}=0, q_{1}=q_{2}=2, d_{1}=0.2, d_{2}=0.4, T=4, S(0)=I(0)=y(0)=2, \alpha=0.55$.
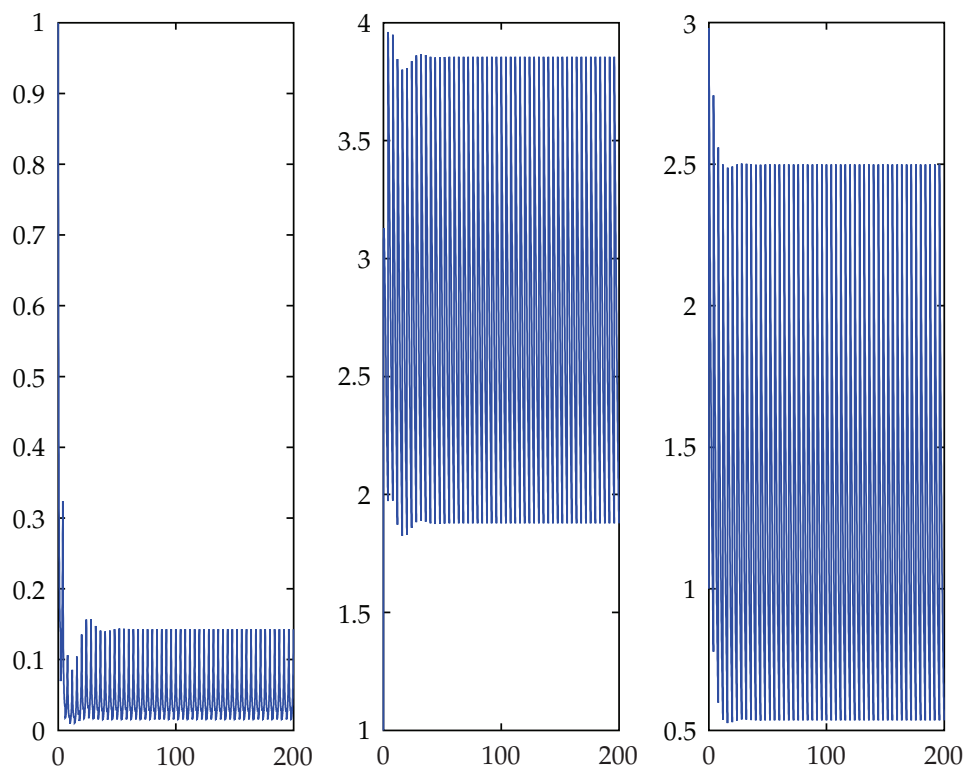

$-y(t)$

Figure 2: Permanence of (1.2) with $r=4, k=8, a=2, c=0.5, p_{1}=0.5, p_{2}=p_{3}=0, q_{1}=q_{2}=2, d_{1}=0.2$, $d_{2}=0.4, T=4, S(0)=I(0)=y(0)=2, \alpha=0.4$. 


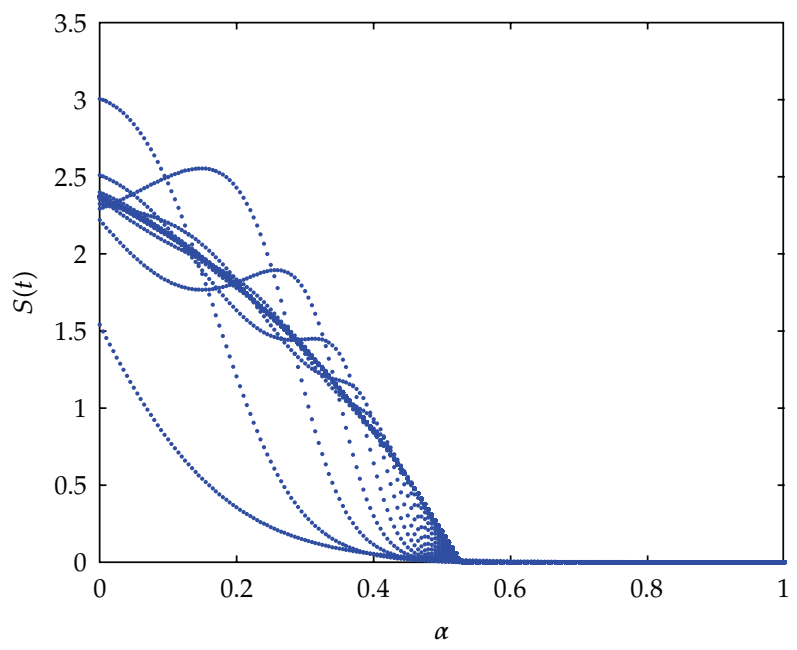

Figure 3: Bifurcation diagrams of susceptible pest for (1.2) with $r=4, k=8, a=2, c=0.5, p_{1}=0.5, p_{2}=$ $p_{3}=0, q_{1}=q_{2}=2, d_{1}=0.2, d_{2}=0.4, T=4, S(0)=I(0)=y(0)=2$. For each value of $\alpha$, system (1.2) is integrated over 500 pulsing cycles, and the stroboscopic measurements of $S(t)$ are plotted, $\alpha$ is over $[0,1]$.

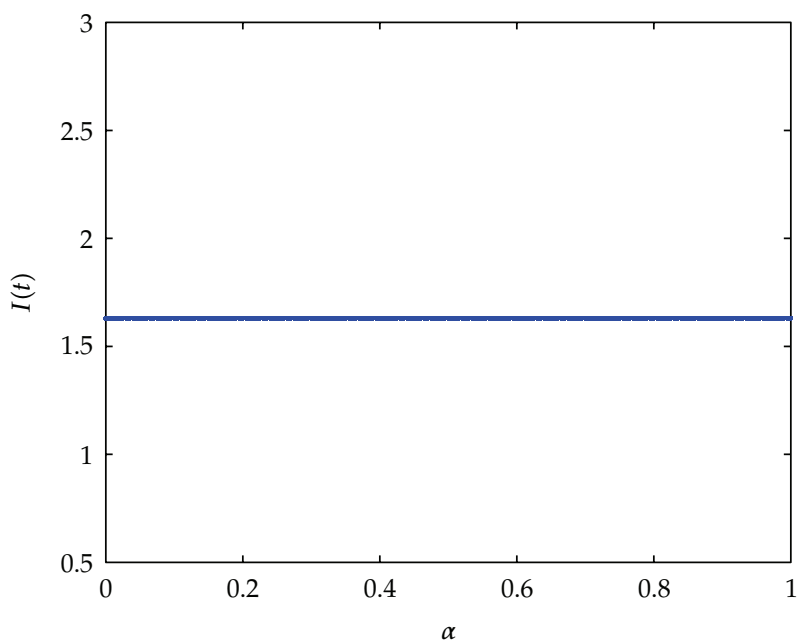

Figure 4: Bifurcation diagrams of infective pest for (1.2) with $r=4, k=8, a=2, c=0.5, p_{1}=0.5, p_{2}=p_{3}=0$, $q_{1}=q_{2}=2, d_{1}=0.2, d_{2}=0.4, T=4, S(0)=I(0)=y(0)=2$. For each value of $\alpha$, system (1.2) is integrated over 500 pulsing cycles, and the stroboscopic measurements of $I(t)$ are plotted, $\alpha$ is over $[0,1]$.

periodic pesticide spraying on all species and periodically releasing predator at fixed different time. Conditions ensuring the existence and global stability of the extinction of susceptible prey as well as the permanence of this system are established.

Our results show that the susceptible prey is either extinct or permanent, that is, when the stability of extinct equilibrium is lose, the system becomes permanent. We conclude that, to reduce pest population, a strategy should aim to make the inequality in Theorem 3.2 hold. This can be done by increasing the value of parameters $\alpha, T$, and $p_{1}$ or decreasing the value of parameters $q_{1}$ and $q_{2}$, that is, releasing more infective pests, using more effective pesticides 


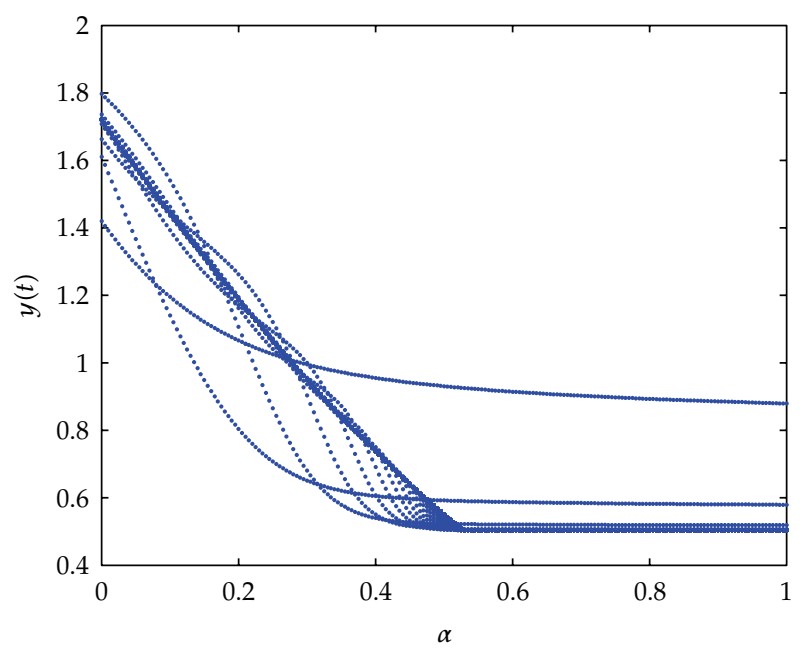

Figure 5: Bifurcation diagrams of predator for (1.2) with $r=4, k=8, a=2, c=0.5, p_{1}=0.5, p_{2}=p_{3}=0$, $q_{1}=q_{2}=2, d_{1}=0.2, d_{2}=0.4, T=4, S(0)=I(0)=y(0)=2$. For each value of $\alpha$, system (1.2) is integrated over 500 pulsing cycles, and the stroboscopic measurements of $y(t)$ are plotted, $\alpha$ is over $[0,1]$.

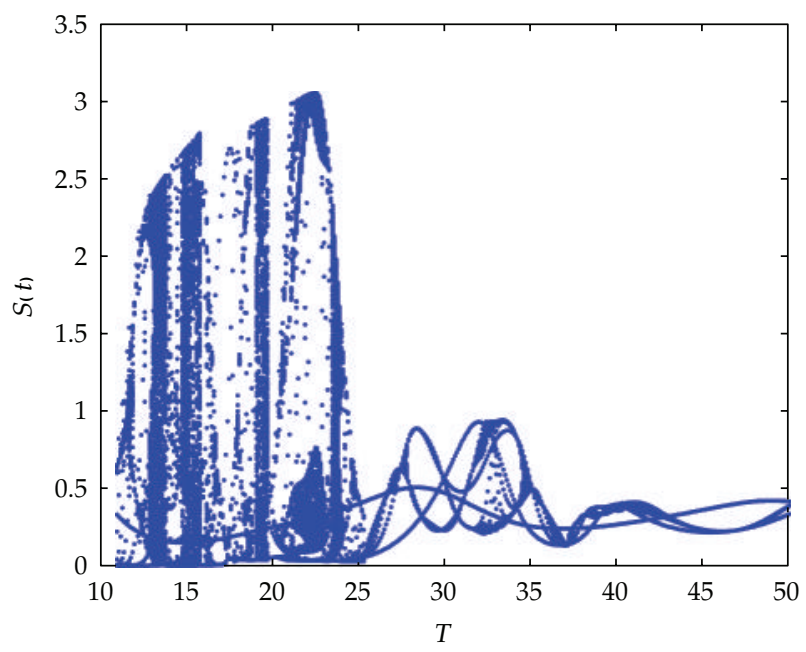

Figure 6: Bifurcation diagrams of susceptible pest for (1.2) with $r=2, K=4, a=2, c=0.5, \alpha=0.6, p_{1}=0.5$, $p_{2}=0.3, p_{3}=0.6, \sigma=0.3, \iota=0.5, d_{1}=0.2, d_{2}=0.4, q_{1}=2, q_{2}=4, S(0)=I(0)=y(0)=1$. For each value of $T$, system (1.2) is integrated over 400 pulsing cycles, and the last 400 stroboscopic measurements of $S(t)$ are plotted, and $T$ is over $[10,50]$.

for the prey and increasing numbers of releasing of infective prey and predator (the natural enemy of the pest). By numerical analysis, small changes of parameters $\alpha, T, p_{i}$, or $q_{i}(i=1,2)$ may make much difference on the dynamics of (1.2). This indicates that pest control strategy is a very complex issue, and integrated pest management may be more effective than those when only spraying pesticides is used or only infective pest is released or only natural enemy is released. Hence, integrated pest management may be a prior strategy for pest management.

Finally, many authors [15, 29-31] pay much attention to study the existence of epidemic equilibrium points, dynamical effects from delays, and stochastic impulse in recent 


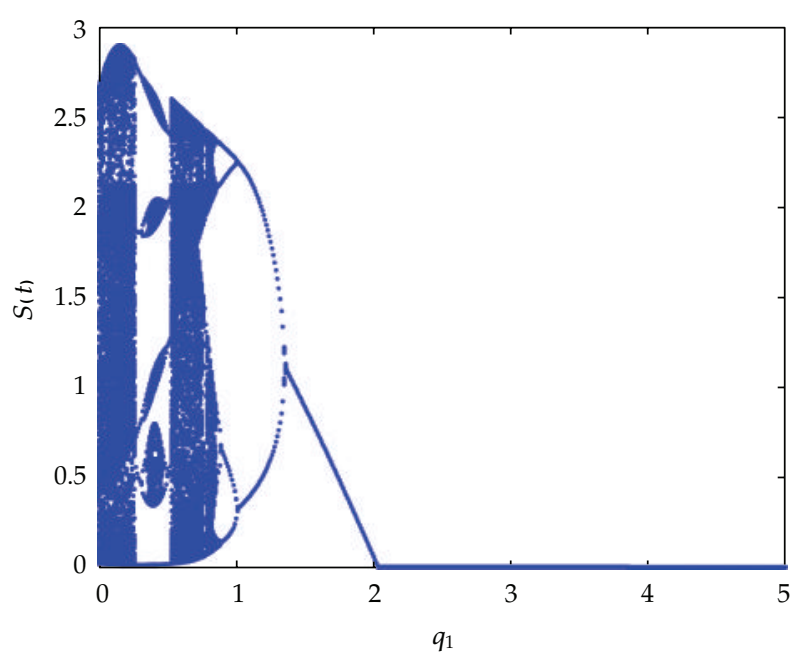

Figure 7: Bifurcation diagrams of susceptible pest for (1.2) with $r=2, K=4, a=2, c=0.5, \alpha=0.6, p_{1}=0.5$, $p_{2}=0.3, p_{3}=0.6, \sigma=0.3, \iota=0.5, d_{1}=0.2, d_{2}=0.4, q_{2}=4, T=10, S(0)=I(0)=y(0)=1$. For each value of $T$, system (1.2) is integrated over 500 pulsing cycles, and the last 400 stroboscopic measurements of $S(t)$ are plotted, and $q_{1}$ is over $[0,5]$.

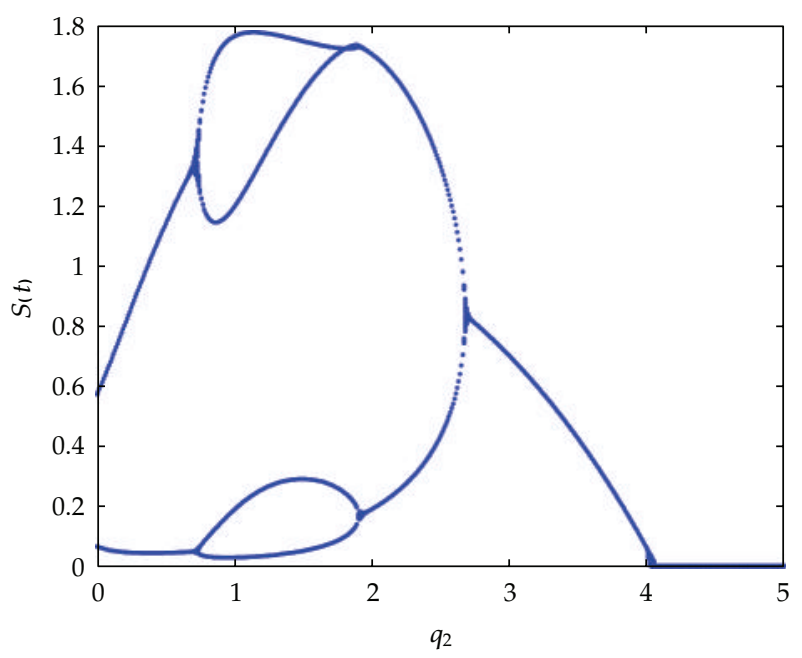

Figure 8: Bifurcation diagrams of susceptible pest for (1.2) with $r=2, K=4, a=2, c=0.5, \alpha=0.6, p_{1}=0.5$, $p_{2}=0.3, p_{3}=0.6, \sigma=0.3, \iota=0.5, d_{1}=0.2, d_{2}=0.4, q_{1}=2, T=10, S(0)=I(0)=y(0)=1$. For each value of $T$, system (1.2) is integrated over 800 pulsing cycles, and the last 400 stroboscopic measurements of $S(t)$ are plotted, and $q_{2}$ is over $[0,5]$.

years. Then for (1.2), how about the potential presence or not of epidemic equilibrium points of the prey and the possible extensions to the incorporation of delays? On the other hand, if the predator is released stochastically, how does it affect the dynamics of (1.2)? we leave these interesting problems as our future work. 


\section{Acknowledgments}

The authors would like to thank the reviewers for their valuable comments and constructive suggestions, which are very useful for improving the quality of this paper. The paper is supported by National Natural Science Foundation of China (11161015) and Doctoral Foundation of Guilin University of Technology (2010).

\section{References}

[1] H. K. Baek, S. D. Kim, and P. Kim, "Permanence and stability of an Ivlev-type predator-prey system with impulsive control strategies," Mathematical and Computer Modelling, vol. 50, no. 9-10, pp. 13851393, 2009.

[2] J. J. Jiao, X. S. Yang, S. Cai, and L. S. Chen, "Dynamical analysis of a delayed predator-prey model with impulsive diffusion between two patches," Mathematics and Computers in Simulation, vol. 80, no. 3, pp. 522-532, 2009.

[3] K. Y. Liu, X. Z. Meng, and L. S. Chen, "A new stage structured predator-prey Gomportz model with time delay and impulsive perturbations on the prey," Applied Mathematics and Computation, vol. 196, no. 2, pp. 705-719, 2008.

[4] M. X. Liu, Z. Jin, and M. Haque, "An impulsive predator-prey model with communicable disease in the prey species only," Nonlinear Analysis. Real World Applications, vol. 10, no. 5, pp. 3098-3111, 2009.

[5] X.-Z. Meng, L.-S. Chen, and Q.-X. Li, "The dynamics of an impulsive delay predator-prey model with variable coefficients," Applied Mathematics and Computation, vol. 198, no. 1, pp. 361-374, 2008.

[6] Y. F. Shao and B. X. Dai, "The dynamics of an impulsive delay predator-prey model with stage structure and Beddington-type functional response," Nonlinear Analysis. Real World Applications, vol. 11, no. 5, pp. 3567-3576, 2010.

[7] Y. F. Shao, B. X. Dai, and Z. G. Luo, "The dynamics of an impulsive one-prey multi-predators system with delay and Holling-type II functional response," Applied Mathematics and Computation, vol. 217, no. 6, pp. 2414-2424, 2010.

[8] Y. F. Shao, "Analysis of a delayed predator-prey system with impulsive diffusion between two patches," Mathematical and Computer Modelling, vol. 52, no. 1-2, pp. 120-127, 2010.

[9] R. Q. Shi, X. Jiang, and L. S. Chen, "A predator-prey model with disease in the prey and two impulses for integrated pest management," Applied Mathematical Modelling, vol. 33, no. 5, pp. 2248-2256, 2009.

[10] X. Y. Song and Z. Y. Xiang, "The prey-dependent consumption two-prey one-predator models with stage structure for the predator and impulsive effects," Journal of Theoretical Biology, vol. 242, no. 3, pp. 683-698, 2006.

[11] H. Su, B. X. Dai, Y. M. Chen, and K. W. Li, "Dynamic complexities of a predator-prey model with generalized Holling type III functional response and impulsive effects," Computers $\mathcal{E}$ Mathematics with Applications, vol. 56, no. 7, pp. 1715-1725, 2008.

[12] W. M. Wang, H. Wang, and Z. Li, "The dynamic complexity of a three-species Beddington-type food chain with impulsive control strategy," Chaos, Solitons and Fractals, vol. 32, no. 5, pp. 1772-1785, 2007.

[13] X. Q. Wang, W. M. Wang, and X. L. Lin, "Dynamics of a two-prey one-predator system with Watttype functional response and impulsive control strategy," Chaos, Solitons and Fractals, vol. 40, no. 5, pp. 2392-2404, 2009.

[14] W. M. Wang, X. Q. Wang, and Y. Z. Lin, "Complicated dynamics of a predator-prey system with Watttype functional response and impulsive control strategy," Chaos, Solitons and Fractals, vol. 37, no. 5, pp. 1427-1441, 2008.

[15] M. De la Sen, R. P. Agarwal, A. Ibeas, and S. Alonso-Quesada, “On a generalized time-varying SEIR epidemic model with mixed point and distributed time-varying delays and combined regular and impulsive vaccination controls," Advances in Difference Equations, vol. 2010, Article ID 281612, 42 pages, 2010.

[16] L. S. Chen, X. Y. Song, and Z. Lu, Mathematical Models and Methods in Ecology, Sichuan Technology Publishing Company, Chengdu, China, 2003.

[17] J. Cost, Comparing predator-prey models qualitatively and quantitatively with ecological time-series data [Ph.D. thesis], Institute National Agronomique, Paris, France, 1998.

[18] Z. E. Ma, Mathematical Modelling and Study of Species Ecology, Anhui Education Publishing Company, Hefei, China, 1996. 
[19] J.-W. Feng and S.-H. Chen, "Global asymptotic behavior for the competing predators of the Ivlev types," Mathematica Applicata, vol. 13, no. 4, pp. 85-88, 2000.

[20] L. Ling and W. M. Wang, "Dynamics of a Ivlev-type predator-prey system with constant rate harvesting," Chaos, Solitons and Fractals, vol. 41, no. 4, pp. 2139-2153, 2009.

[21] X. J. Wu and W. T. Huang, "Dynamics analysis of a one-prey multi-predator impulsive system with Ivlev-type functional," Ecological Modelling, vol. 220, pp. 774-783, 2009.

[22] H. D. Burges and N. Hussey, Microbial Control of Insect and Mites, Academic Press, New York, NY, USA, 1971.

[23] L. A. Falcon, "Problem associated with the use of arthropod viruses in pest control," Annual Review of Entomology, vol. 21, pp. 305-324, 1976.

[24] P. Georgescu and H. Zhang, "An impulsively controlled predator-pest model with disease in the pest," Nonlinear Analysis. Real World Applications, vol. 11, no. 1, pp. 270-287, 2010.

[25] B. Liu, Y. Zhang, and L. Chen, "The dynamical behaviors of a Lotka-Volterra predator-prey model concerning integrated pest management," Nonlinear Analysis. Real World Applications, vol. 6, no. 2, pp. 227-243, 2005.

[26] R. L. Doutt and P. DeBach, Biological Control of Insect Pests and Weeds, Reinhold Publishing Corporation, New York, NY, USA, 1964.

[27] D. Bainov and P. Simeonov, Impulsive Differential Equations: Periodic Solution and Applications, Longman scientific Technical, Harlow, UK, 1993.

[28] V. Laksbmikanham, D. Bainov, and P. Simeonov, Theory of Impulsive Differential Equations, World Scientific Publisher, Singapore, 1989.

[29] M. De la Sen, A. Ibeas, S. Alonso-Quesada, and R. Nistal, "On the equilibrium points, boundedness and positivity of a sveirs epidemic model under constant regular constrained vaccination," Informatica, vol. 22, no. 3, pp. 339-370, 2011.

[30] H.-F. Huo and Z.-P. Ma, "Dynamics of a delayed epidemic model with non-monotonic incidence rate," Communications in Nonlinear Science and Numerical Simulation, vol. 15, no. 2, pp. 459-468, 2010.

[31] I. A. Dzhalladova, J. Baštinec, J. Diblík, and D. Y. Khusainov, "Estimates of exponential stability for solutions of stochastic control systems with delay," Abstract and Applied Analysis, vol. 2011, Article ID 920412, 14 pages, 2011. 


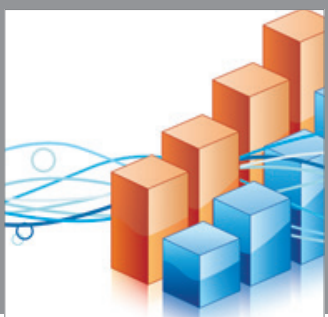

Advances in

Operations Research

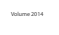

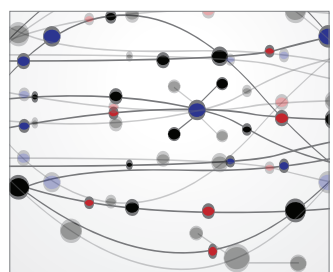

\section{The Scientific} World Journal
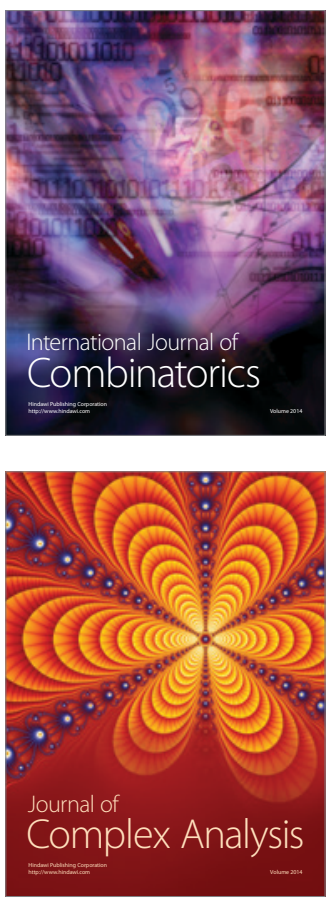

International Journal of

Mathematics and

Mathematical

Sciences
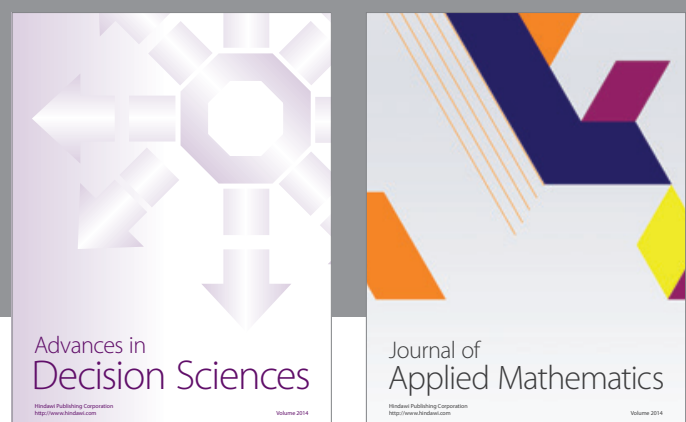

Journal of

Applied Mathematics
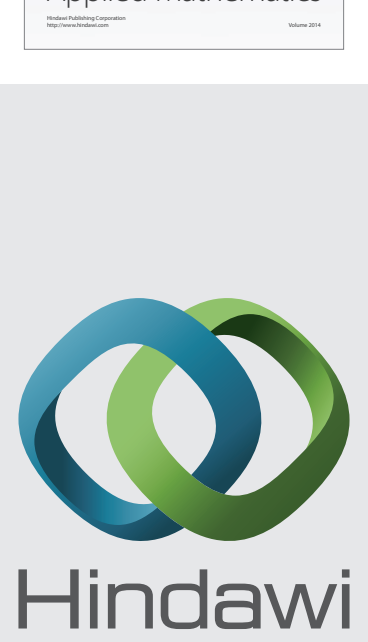

Submit your manuscripts at http://www.hindawi.com
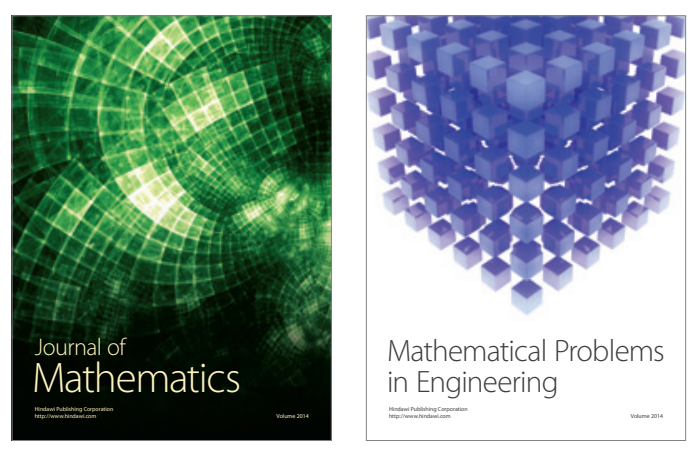

Mathematical Problems in Engineering
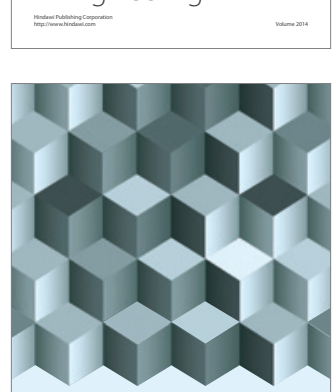

Journal of

Function Spaces
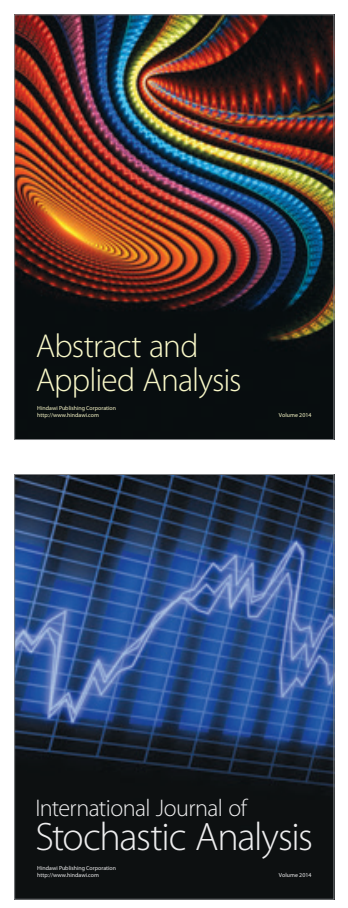

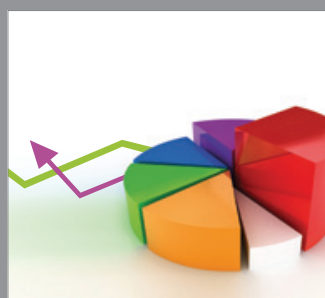

ournal of

Probability and Statistics

Promensencen
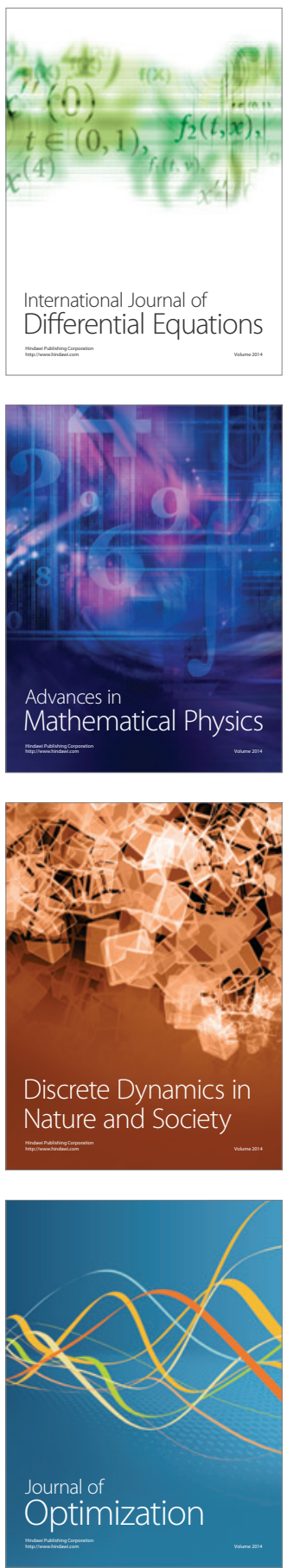